\title{
A Review on Optical Fiber Long Period Grating, its applications in Optical Communication System
}

\author{
Mr.Puneet Sehgal ${ }^{1}$, Ms Himani Dua ${ }^{2}$ \\ Department of Electronics, Assistant Professor, A.R.S.D. College, University of Delhi ${ }^{1}$ \\ Department of Instrumentation, Assistant Professor, Shaheed Rajguru College of Applied Sciences for Women, \\ University of Delhi ${ }^{2}$
}

\begin{abstract}
Innovations in optical fiber technology are revolutionizing world communications. As we can se that optical fiber long period grating can be used in designing of devices which are used to meet the growing demands for various ranges in the field of optical communication systems. Thus, this paper deals with the descriptive study of long period fiber grating (LPG) and its applications in emerging field of optical communication systems. LPG forms an important component of optical communication. The paper covers the analysis of long fiber grating and their fabrication. This paper also deals with the cladding mode analysis of the fiber which describes the inaccuracies of two layer model of the fiber and implementation of three layer fiber geometry to calculate the effective refractive index of the fiber so that it can effectively couple the signal for its efficient transmission. In the cladding mode analysis the whole mathematical derivation along with the requisite mathematical expressions are explained to find the effective refractive indices of the various cladding modes being supported by the fiber which are used to plot the transmission spectrum of the LPG designed for a particular frequency or wavelength used for telecommunication purposes.
\end{abstract}

Keywords: Optical Fiber Communication, long period Grating, Optical Filter, coupled mode equations, LPG fabrication.

\section{INTRODUCTION}

The rapid progress and development in optical fiber communication and sensing systems have impulsively speed up the exploration of researchers and scientists to improve and develop the optical devices, which could overcome the existing limitations and perform the task of precursors for future advancements in photonics technology.

Optical fiber can be used as sensors to measure strain, temperature, pressure and other quantities by modifying a fiber so that the quantity to be measured modulates the intensity, phase, polarization, wavelength or transit time of light in the fiber. Sensors that vary the intensity of light are the simplest, since only a simple source and detector are required. A particularly useful feature of such fiber optic sensors is that they can, if required, provide distributed sensing over distances of up to one meter. Optical Communication systems based on Wavelength Division Multiplexing, $\mathrm{N}$ signal laser beams at $\mathrm{N}$ different wavelengths (each carrying modulated user information) are coupled into the EDFA and propagate down the fiber along with the pump. Gain of the erbium doped fiber amplifier across the total spectrum in use, has emerged as topic of research in recent years. LPG'S have also been used as gain flattening filters for Erbium doped Fiber amplifiers [1], and as optical fiber polarizer's[2] because of their low insertion loss and low back reflections which in addition to gain equalization; give an overall gain enhancement as well as Amplified Spontaneous Emission (ASE) noise suppression. The development of fiber gratings had a significant impact on research and development in telecommunications and fiber optic sensing. Fiber gratings are made by introducing periodic perturbation in their refractive index .Fiber gratings are intrinsic devices that allow control over the properties of light propagating within the fiber-they are used as spectral filters, as dispersion compensating components and in wavelength division multiplexing systems. The sensitivity of their properties to perturbation of the fiber by the surrounding environmental conditions has led to extensive study of their use as fiber sensor elements. Fiber gratings consist of a periodic perturbation of the properties of the optical fiber, generally of the refractive index of the core[3].

\section{PHYSICAL MODEL TO ANALYSE LONG PERIOD GRATING (LPG)}

The long-period grating (LPG) has a period typically in the range $100 \mu \mathrm{m}$ to $1 \mathrm{~mm}$, as illustrated in fig 1[3]. It couples light from a fundamental guided core mode into 
co-propagating cladding modes at various wavelengths, was first reported by Vengsarkar and co-workers in1996 [4]. The high attenuation of the cladding modes results in the transmission spectrum of the fiber containing a series of attenuation bands centred at discrete wavelengths, each attenuation band corresponding to the coupling to a different cladding mode and the exact form of the spectrum, and the centre wavelengths of the attenuation bands, are sensitive to the period of the LPG, the length of the LPG (typically of the order of $30 \mathrm{~mm}$ ) and to the local environment: temperature, strain, bend radius and to the refractive index of the medium surrounding the fiber,

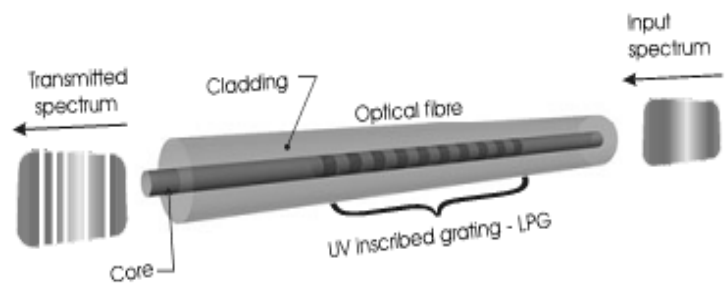

Fig1: Schematic of an LPG [3]

Thus Changes in these parameters can modify the period of the LPG and differential refractive index of the core and cladding modes which modifies the phase matching conditions for coupling to the cladding modes and results in change in the central wavelength of the attenuation bands [3]. The sensitivity to a particular measure is dependent upon the composition of the fiber, order of the cladding mode to which the guided optical power is coupled and is different for each attenuation band [3]. This range of responses makes them particularly attractive for sensor applications, with the prospect for multi-parameter sensing using a single sensor element [5]

The LPG couples light from the forward propagating core mode, i.e., the mode guided by the core cladding interface of the fiber to the cladding modes, i.e., the modes guided by the cladding ambient interface of the fiber. As we know that cladding mode is essentially the mode associated with the fiber with a very large core radius $(\sim 62.5 \mu \mathrm{m})$ with the ambient as cladding. The cladding modes can be quickly attenuated. The principle of operation of LPG is similar to that of an FBG, except that in long period grating, the incident light traveling in the core mode excites the cladding guided modes at each perturbation as shown in Fig 2. Light coupled to the $m^{\text {th }}$ cladding mode at interface 1 travels with the propagation constant $\beta_{c l}^{m}$ and interferes with the light coupled to the $m^{\text {th }}$ cladding mode at interface 2 . The light coupled at interface 2 has an additional phase due to the core mode having traversed a length $\Lambda$ with the propagation constant $\beta_{c o}$ corresponding to the core mode. Hence, the phase difference between two waves is $\Delta \phi=\left(\beta_{c o}-\beta_{c l}^{m}\right) \Lambda$ and to undergo constructive interference the phase difference between them should be an integral multiple of $2 \pi$. This condition of constructive interference leads to the phase matching condition between core guided and a cladding mode of $\mathrm{m}^{\text {th }}$ order and can be expressed as

$\Delta \phi=\left(\beta_{c o}-\beta_{c l}^{m}\right) \Lambda=\frac{2 \pi}{\lambda_{0}}\left(n_{\text {eff }}^{c o}-n_{\text {eff }}^{c l, m}\right) \Lambda=2 \pi$

Where:

$\beta_{c o}=$ propagation constant of the core

$\beta_{c l}^{m}=$ propagation constant of the cladding at $\mathrm{m}^{\text {th }}$ order

$\mathrm{n}^{\mathrm{co}}{ }_{\text {eff }}=$ effective index of the core

$\mathrm{n}^{\mathrm{cl}, \mathrm{m}}{ }_{\text {eff }}=$ effective index of the $\mathrm{nth}$ cladding mode

$\Lambda=$ LPG period, which is much longer for co propagating coupling at a given wavelength than for the counter propagating coupling.

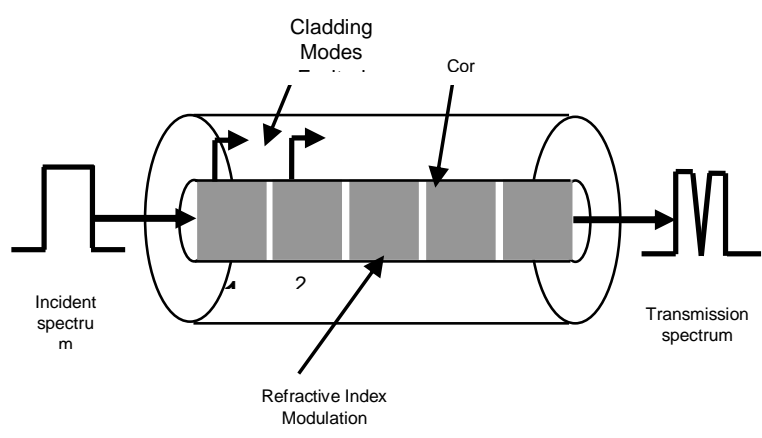

Fig2: A typicaı scnemauc of a Long Period Grating

\section{PRINCIPLE OF OPERATION}

As mentioned earlier a long period fiber grating is formed typically by introducing a periodic refractive index modulation in the core of the optical fiber. LPG couples an incident fundamental core mode $\left(L P_{01}\right)$ to forward propagating cladding modes $\left(L P_{0 \mathrm{~m}}\right)$ when the phase matching conditions are satisfied. The cladding modes are lossy and can be easily attenuated by introducing a bend. As a result the conventional transmission spectra of an LPG consists of a series of attenuation bands, each corresponding to coupling from the propagating core mode to a particular cladding mode[6]. Defining $\Delta \beta$ as the difference between the two propagation constants $\beta_{c o}$ and $\beta_{c l}^{m}$ (where $\beta_{c o}$ denotes the propagation constant of the fundamental $\mathrm{LP}_{01}$ core mode and the propagation 
constants of the cladding modes given by $\beta_{c l}^{m}$ where the superscript denotes the order of the mode), i.e., $\Delta \beta=\left(\beta_{c o}-\beta_{c l}^{m}\right)$, the phase matching condition equation can be written as $\Delta \beta=K=\frac{2 \pi}{\Lambda}$ in terms of $\Delta \beta$ and grating period $\Lambda$ where $K$ is known as the grating vector. The attenuation notches appear in the transmission spectrum of LPG at wavelength $\lambda_{0}$ where the core mode and a particular cladding mode is phase matched. since $\beta=\frac{2 \pi}{\lambda_{0}} n_{\text {eff }}$, the phase matching condition in terms of the coupling wavelength $\lambda_{0}$ can be expressed as[3][6]

$$
\lambda_{0}=\left(n_{e f f}^{c o}-n_{e f f}^{c l, \mathrm{~m}}\right) \Lambda
$$

The relative positions of the propagation constants $\beta_{c l}^{m}$ of the cladding modes are shown with respect to the propagation constant $\beta_{c o}$ of the core mode[3] [6]. There is a purely sinusoidal index modulation along the fiber with the periodic index structure being perpendicular to the fiber axis. These assumptions exclude blazed gratings and as a result modal overlap conditions dictate that the fundamental guided mode can couple only to those cladding modes that are azimuthally symmetric. Thus as order of mode coupling $\mathrm{m}$ increases grating period $\Lambda$ decreases[35].
The axis of the figure represents the $\beta$-axis in Fig 3.The open circle represents the core guided fundamental mode for $n_{2}<n_{\text {eff }}<n_{1}$; filled circles represent cladding modes for which $n_{a}<n_{\text {eff }}<n_{2}$. The hatched region represents the continuum of radiation modes for which $n_{\text {eff }}<n_{a}$. Hence, the guided core mode is phase matched to a co-propagating $L P_{02}$ cladding mode, for a grating of period $\Lambda=\frac{2 \pi}{\Delta \beta 2}$. Similarly, the guided core mode becomes phase matched to a co-propagating $L P_{03}$ cladding mode for a grating of period $\Lambda=\frac{2 \pi}{\Delta \beta_{1}}$. The pictorial description implies that for a given $\lambda_{0}$, depending on the periodicity $\Lambda$ one can induce mode coupling between the fundamental core mode and several different cladding modes.Since in LPG $\Delta \beta=\left(\beta_{c o}-\beta_{c l}^{m}\right)$ decreases as modes increases[6].

\section{IV.EFFECT OF GRATING PERIOD AND RESONANCE WAVELENGTHS}

To generate phase matching curves one requires the propagation constants of the guided core mode and a given cladding mode of the fiber at different wavelengths. From these we obtain periodicities $\Lambda$ that meet the phase matching condition given by equation, $\Lambda=\frac{\lambda_{0}}{\left(n_{e f f}^{c o}-n_{e f f}^{c l, m}\right)}[3][6]$

The choice of grating period, $\Lambda$ also allows the designer to vary the separation between the resonance wavelengths, $\delta \lambda$. We now try to estimate the wavelength spacing between resonance wavelengths corresponding to coupling to the two adjacent cladding modes for a given periodicity $\Lambda$ thus phase matching conditions for $m^{\text {th }}$ and $(m+1)^{\text {th }}$ cladding mode can be expressed as

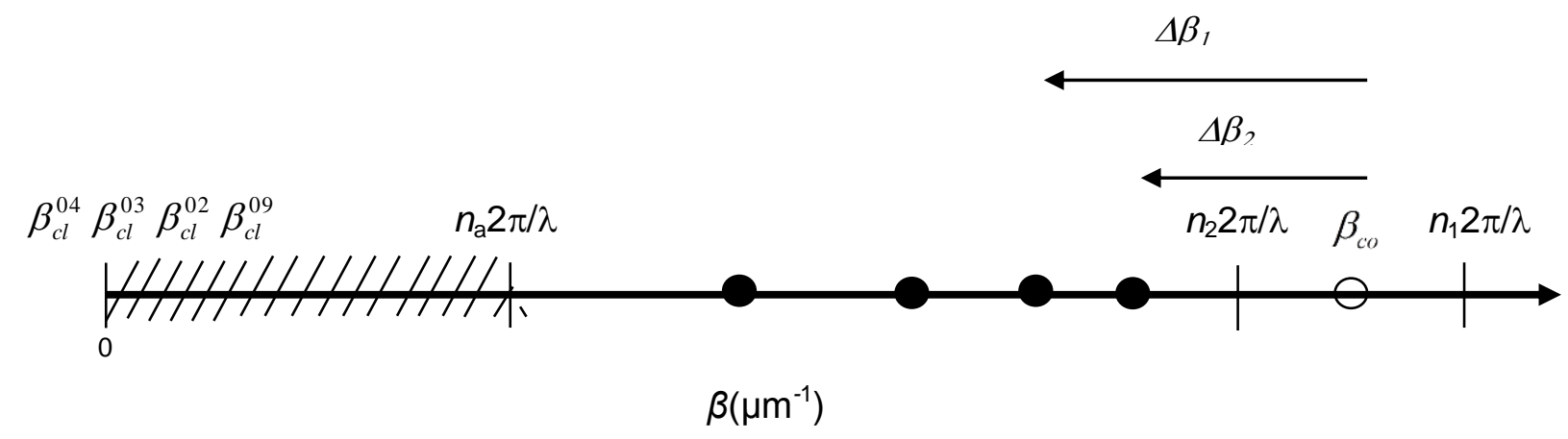

Fig3: $\beta$ - plot for mode-coupling mechanism in long period grating. 


$$
\begin{aligned}
& \lambda_{\mathrm{m}}=\left(n_{e f f}^{c o}-n_{e f f}^{c l, \mathrm{~m}}\right) \Lambda \text { and } \\
& \lambda_{\mathrm{m}+1}=\left(n_{e f f}^{c o}-\boldsymbol{n}_{e f f}^{c l, \mathrm{~m}+1}\right) \Lambda
\end{aligned}
$$

Hence, the wavelength spacing $\delta \lambda$ between the two resonant wavelengths is given by [6]

$$
\delta \lambda=\lambda_{\mathrm{m}+1}-\lambda_{\mathrm{m}}=\left(n_{e f f}^{c l, \mathrm{~m}}-n_{e f f}^{c l, \mathrm{~m}+1}\right) \Lambda
$$

it can be seen that the grating period decreases as coupling between core mode and various cladding modes increases. Theoretically each cladding mode exhibits a turning point. A small reduction in grating period would results in a large change in resonant wavelengths. a slight increase in the grating period would result in no resonant wavelength around turning point. thus the turning point determine the condition of maximum sensitivity for each cladding mode. for long period grating, the region with $\mathrm{d} \lambda / \mathrm{d} \Lambda>0$ with periods to the order of hundreds of micrometers. on the other hand the region with $\mathrm{d} \lambda / \mathrm{d} \Lambda<0$ requires much smaller grating periods in fiber bragg gratings[6].

\section{V.GRATING FABRICATION}

The fabrication of Fiber gratings relies upon the introduction of a periodic modulation of the optical properties of the fiber. This may be achieved by permanent modification of the refractive index of the core of the optical fiber or by physical deformation of the fiber[3]. The modulation of the core refractive index has been achieved by ultraviolet (UV) irradiation[7]-[10], ion implantation[11], and irradiation by femtosecond pulses in the infrared[12], irradiation by $\mathrm{CO}_{2}$ lasers[13-14], diffusion of dopants into the core[15-16], relaxation of mechanical stress[17] and electrical discharges[18-19]. The deformation of the fiber has been achieved mechanically[20-21], by tapering the fiber[22] or by deformation of the core[23-24] or cladding[25].

\section{LPG FABRICATION}

LPGs have been fabricated in photonic crystal fiber by periodically collapsing the holes of the fiber using $\mathrm{CO}_{2}$ laser heat treatment[26]. The UV-induced index modulation is typically achieved in Ge-doped silica fibers using wavelengths between 193 and $266 \mathrm{~nm}$ [27]. This method employs the Fabrication of LPG'S. The refractive index change is associated with the formation of $\mathrm{Ge}$ related glass defects. Fibers with high photosensitivity have been developed by co-doping the core with boron and germanium[28] and by hydrogen loading[29]. The hydrogen loading treatment is used in optical fiber to increase its photosensitivity. The refractive index modulation may be built up on a point-by-point basis-a very flexible technique - or the entire length of the LPG may be formed simultaneously by exposure of the fiber though an amplitude mask[30], via a patterned mirror[31] or using a micro lens array[32] —facilitating rapid and reproducible LPG fabrication.

The use of UV exposure is well established, due to its widespread use in the fabrication of FBGs. Its use has implications for the spectral characteristics and stability of the LPG spectrum. The diffusion of hydrogen from unexposed to exposed regions then acts to increase the amplitude of the refractive index modulation, as the refractive index increases further in the exposed regions (increasing hydrogen concentration) and decreases in the unexposed region (decreasing hydrogen concentration). Thus the strength of the LPG grows. This is followed by a slow out diffusion whereby the refractive index of the core decreases, causing a decrease in wavelength.

The technique of UV exposure is widely used in the fabrication of LPG'S, as it stabilizes the spectrum of LPG. To increase the modulation in the refractive index.The hydrogen gas is diffused from unexposed UV region to exposed UV area. Hence the refractive index increases in the exposed region while decreases at the other. Then slow diffusion process is carried out to decrease the refractive index of the core as the wavelength decreases.

For telecommunication areas use, LPG'S are fabricated at wavelengths $1300 \mathrm{~nm}$ and $1550 \mathrm{~nm}$ and the spectrum is monitored by coupling light from super fluorescent fiber sources or super luminescent diodes in the fiber and recording the transmission using an optical spectrum analyzer. There have been reports of the use of fibers with lower cutoff wavelengths, $650 \mathrm{~nm}$, allowing the operation of the LPG within the response of silicon detectors, facilitating the use of low-cost CCD spectrometers [33].

\section{CLADDING (COUPLED) MODE ANALYSIS}

\section{Cladding modes}

Cladding modes are those modes which are propagated by total internal reflection at the cladding air interface. Calculation of cladding modes uses the approximation that the fiber can be considered as a multimode step index structure ignoring the presence of core, an approach that has commonly been used to simplify cladding mode analysis[34].

\section{Two Layer Model}

The two-layer model basically treats the cladding and core as one multimode fiber and the surrounding environment as the new cladding only in the LPG region[34].

\section{Three layer model}

To calculate $\mathrm{n}^{\mathrm{cl}}$ eff the three layer model is used to overcome the design inaccuracies of two layer model. In three layer model the variation of effective refractive 
index can be calculated correctly up to fourth decimal point whereas this is not possible in two layer model[34].

The complete refractive index profile for evaluating the propagation characteristics of both core and cladding modes is given by [34]

$$
\begin{aligned}
n & =n_{1} & & r<a_{c o} \\
& =n_{2} & & a_{c o}<r<a_{c l} \\
& =n_{a} & & r>a_{c l}
\end{aligned}
$$

Where symbols have their usual meanings. The crosssection view can be shown as

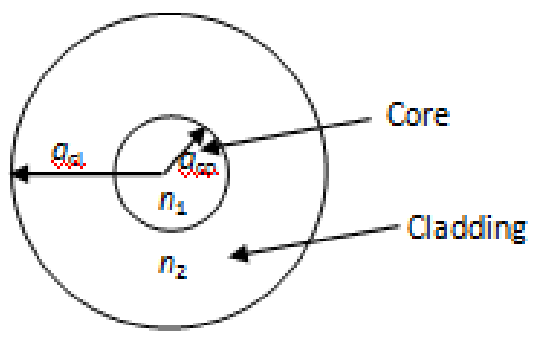

D.

Ambient

Fig 4:Complete Three-Layer Fiber Geometry for calculation of propagation constants of different modes

The differential equations for determining the radial variation of field $R(r)$ are of the form given in equations. For the symmetric $(l=0)$ core guided mode ( $n_{1}>\beta / k_{0}>n_{2}$ ) the equations reduce to

$$
\begin{array}{cc}
\frac{d^{2} R}{d r^{2}}+\frac{1}{r} \frac{d R}{d r}+\left(\frac{U^{2}}{a_{c o}^{2}}\right) R=0 & r<a_{c o} \\
\frac{d^{2} R}{d r^{2}}+\frac{1}{r} \frac{d R}{d r}-\left(\frac{W^{2}}{a_{c o}^{2}}\right) R=0 & a_{c o}<r<a_{c}
\end{array}
$$$$
\frac{d^{2} R}{d r^{2}}+\frac{1}{r} \frac{d R}{d r}-\left(\frac{W_{l}^{2}}{a_{c o}^{2}}\right) R=0
$$

where $U=a_{c o} k_{0} \sqrt{\left(n_{1}^{2}-n_{e f f}^{2}\right)}$,

$W=a_{c o} k_{0} \sqrt{\left(n_{e f f}^{2}-n_{2}^{2}\right)}$ and

$$
W_{1}=a_{c o} k_{0} \sqrt{\left(n_{e f f}^{2}-n_{a}^{2}\right)} \text {. }
$$

The solutions to above equations are

$$
\begin{aligned}
R(r) & =A J_{0}\left(U r / a_{c o}\right) & & r \leq a_{c o} \\
& =B K_{0}\left(W r / a_{c o}\right)+C I_{0}\left(W r / a_{c o}\right) & & a_{c o} \leq r \leq<a_{c l} \\
& =D K_{0}\left(W_{1} r / a_{c o}\right) & & r \geq a_{c l}
\end{aligned}
$$

Similarly for the symmetric cladding modes for which $n_{2}>\beta / k_{0}>n_{a}$, the corresponding equations are

$$
\begin{array}{cc}
\frac{d^{2} R}{d r^{2}}+\frac{1}{r} \frac{d R}{d r}+\left(\frac{U^{2}}{a_{c o}{ }^{2}}\right) R=0 & r<a_{c o} \\
\frac{d^{2} R}{d r^{2}}+\frac{1}{r} \frac{d R}{d r}+\left(\frac{U_{1}^{2}}{a_{c o}{ }^{2}}\right) R=0 & a_{c o}<r<a_{c l}
\end{array}
$$

$$
\frac{d^{2} R}{d r^{2}}+\frac{1}{r} \frac{d R}{d r}-\left(\frac{W_{1}^{2}}{a_{c o}^{2}}\right) R=0 \quad r>a_{c l}
$$

and leads to the following solutions

$$
\begin{aligned}
R(r) & =A_{1} J_{0}\left(U r / a_{c o}\right) & & r \leq a_{c o} \\
& =B_{1} Y_{0}\left(U_{1} r / a_{c o}\right)+C_{1} J_{0}\left(U_{1} r / a_{c o}\right) & & a_{c o} \leq r \leq a_{c l} \\
& =D_{1} K_{0}\left(W_{1} r / a_{c o}\right) & & r \geq a_{c l}
\end{aligned}
$$

where $U_{1}=a_{c o} k_{0} \sqrt{\left(n_{2}^{2}-n_{e f f}^{2}\right)} . J_{0}$ and $I_{0}$ are the

(3) Bessel functions and modified Bessel functions of the first kind, while $Y_{0}$ and $K_{0}$ are the Bessel functions and modified Bessel functions of the second kind.

(4) Using the continuity of $R(r)$ at $r=a_{c o}$ and $r=a_{c l}$ and that of $\frac{d R}{d r}$ at $r=a_{c o}$ and $r=a_{c l}$ for the solutions in 

equation (9), the constants $B, C$ and $D$ can be expressed in
terms of $A$ and one obtains an eigenvalue for the where $c=\frac{a_{c l}}{a_{c o}}$. The constant $A$ is obtained from the
propagation constant of the core guided mode as

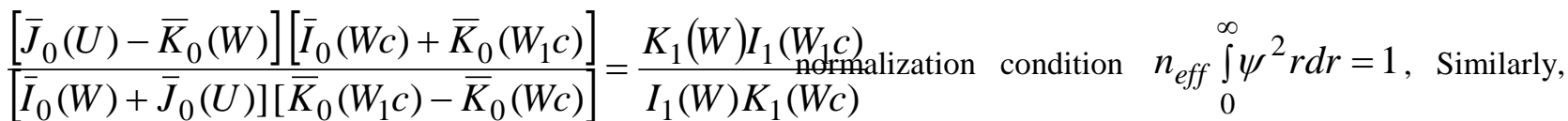

where $\bar{Z}_{m}(x)=\frac{Z_{m}(x)}{x Z_{m+1}(x)} \quad(Z$ represents the Bessel functions $J, I$ and $K$ ). using the continuity of $R(r)$ at $r=a_{c o}$ and $r=a_{c l}$ and that of $\frac{d R}{d r}$ at $r=a_{c o}$ and $r=a_{c l}$ for the solutions in equation (9) we obtain the following eigenvalue equation for the cladding modes

The constants $B, C$ and $D$ in terms of $A$ are obtained as follows:

Using the boundary conditions at $r=a_{c o}$

$$
\frac{\left[J_{0}(U)-J_{0}(\bar{U})\right]\left[\bar{K}_{0}(W c)-\bar{Y}_{0}(\bar{U} c)\right]}{\left[\bar{J}_{0}(U)-\bar{Y}_{0}(\bar{U})\right]\left[\bar{K}_{0}(W c)-\bar{J}_{0}(\bar{U} c)\right]}=\frac{Y_{1}(\bar{U}) J_{1}(\bar{U} c)}{Y_{1}(\bar{U} c) J_{1}(\bar{U})}
$$

$$
A J_{0}(U)=B K_{0}(W)+C I_{0}(W)
$$

$$
A U J_{1}(U)=B W K_{1}(W)-C W I_{1}(W) A_{1}
$$

$B$ and $C$ can be expressed in terms of $A$ as

$$
B_{1}=A_{1} \frac{\pi}{2}\left(U_{1} J_{1}\left(U_{1}\right) J_{0}(U)-U J_{1}(U) J_{0}\left(U_{1}\right)\right)
$$

$$
B=A\left(U J_{1}(U) I_{0}(W)+W J_{0}(U) I_{1}(W)\right) \quad C_{1}=A_{1} \frac{\pi}{2}\left(U_{1} Y_{1}\left(U_{1}\right) J_{0}(U)-U J_{1}(U) Y_{0}\left(U_{1}\right)\right)
$$

(13) Where we have used the identity

$$
\text { where we have used the identity } U_{1} J_{1}\left(U_{1}\right) Y_{0}\left(U_{1}\right)-U_{1} Y_{1}\left(U_{1}\right) J_{0}\left(U_{1}\right)=\frac{2}{\pi}
$$

$$
W K_{0}(W) I_{1}(W)+W K_{1}(W) I_{0}(W)=1
$$

$$
C=A\left(W J_{0}(U) K_{1}(W)-U J_{l}(U) K_{0}(W)\right)
$$

Using the continuity at $r=a_{c l}$ gives $D_{1}$ in terms of $B_{1}$ and $C_{1}$ as

$$
\begin{aligned}
& \text { where we have used the identity } \\
& W I_{0}(W) K_{1}(W)+W I_{1}(W) K_{0}(W)=1
\end{aligned}
$$

$$
D_{1}=\frac{B_{1} J_{0}\left(U_{1} c\right)+C_{1} I_{0}\left(U_{1} c\right)}{K_{0}\left(W_{1} c\right)}
$$

The boundary condition at $r=a_{c l}$ gives $D$ in terms of $B \quad A_{1}$ is again obtained by the normalization condition and $C$

$$
D=\frac{B K_{0}(W c)+C I_{0}(W c)}{K_{0}\left(W_{1} c\right)}
$$

Thus we can calculate the $\mathrm{n}_{\text {eff }}$ of the fiber provided the refractive indices of the core, cladding and their respective radii are given to us. Hence we can verify the refractive index of cladding modes from above equations for a given fiber by this three layer model analysis. This analysis is

(15) further used to plot the transmission spectrum of the LPG studied at a particular wavelength, let say at $1550 \mathrm{~nm}$ 


\section{International Journal of Advanced Research in Computer and Communication Engineering}

Vol. 4, Issue 5, May2015

which is the most common wavelength used for telecommunication purposes.

\section{CONCLUSION}

Thus we have discussed about the long period grating, its fabrication and analysis of cladding modes which are propagating in it, this analysis is used to find the effective refractive indices of the various cladding modes being supported by the fiber which are used to plot the transmission spectrum of the LPG designed for a particular frequency or wavelength used for telecommunication purposes.

\section{ACKNOWLEDGEMENT}

The authors are thankful to University of Delhi, Department of Electronic Science, South Campus and their respective colleges. Thanks are also due to our colleagues, for their constant guidance and unconditional support.

\section{REFERENCES}

[1] Qian J R and Chen H E 1998 Gain flattening fibre filters using phase shifted long period fibre grating 34 1132-3Electron. Lett.

[2] Ortega B, Dong I, Lic W E, Sandro J P, Reekie I, Tsypina S I,Bagratashvile V N and Laming R I 1997 High performance

[3] Stephen W James and Ralph P Tatam, Optical fibre long-period grating sensors: characteristics and application ,INSTITUTE OF PHYSICS PUBLISHING MEASUREMENT SCIENCE AND TECHNOLOGY Meas. Sci. Technol. 14 (2003) R49-R61 PII: S0957-0233(03)55184-0

[4] Vengsarkar A M, Lemaire P J, Judkins J B, Bhatia V, Erdogan T and Sipe J E 1996 Long-period grating asband-rejection filters . 14 5864.J. Light wave Technol.

[5] Bhatia V 1999 Applications of long-period gratings to single and multi-parameter sensing Opt. Express 4 457-66

[6] Optical Fiber Torsion Sensor with Mechanically Induced Long Period Fiber Gratings in Rare-Earth Doped Fibers, Maria Pulido-Navarro, José Álvarez-Chávez1, Daniel Ceballos-Herrera, Ponciano Escamilla-Ambrosio, Optics and Photonics Journal, 2014, 4, 129135Published Online June 2014 in SciRes.http://www.scirp.org/journal/opjhttp://dx.doi.org/10.4236/o pj.2014.46013

[7] Bhatia V and Vengsarkar A M 1996 Optical fibre long-period grating sensors Opt. Lett. 21 692-4

[8] Chen K P, Herman P R, Tam R and Zhang J 2000 Rapid long-period grating formation in hydrogen loaded fibre with $157 \mathrm{~nm}$ F2 laser radiation Electron. Lett.36 2000-1

[9] Guan B-Ou, Tam H-Y, Ho S-L, Liu S-Y and Dong X-Y 2000 Growth of long-period gratings in H2-loaded fibre after $193 \mathrm{~nm} \mathrm{UV}$ inscription IEEE Photon. Technol. Lett. 12642-4

[10] Blows J and Tang D Y 2000 Gratings written with tripled output of Q-switched Nd:YAG laser Electron. Lett 36 1837-9

[11] Fujumaki M, Ohki Y, Brebner J L and Roorda S 2000 Fabrication of long-period optical fibre gratings by use of ion implantation $O p t$. Lett. 25 88-90

[12] Kondo Y, Nouchi K, Mitsuyu T, WatanabeM, Kazansky P and Hirao K 1999 Fabrication of long-period fibre gratings by focused irradiation of infra-red femtosecond laser pulsesOpt. Lett. 24 646-8

[13] Davis D D, Gaylord T K, Glytsis E N, Kosinski S G, Mettler S C and Vengsarkar A M 1998 Long-period fibre grating fabrication with focused CO2 laser beams Electron.Lett.34 302-3

[14] Drozin L, Fonjallaz P-Y and Stensland L K 2000 Long-period fibre gratings written by $\mathrm{CO} 2$ exposure of $\mathrm{H} 2$-loaded standard fibres Electron. Lett.36 742-3
[15] Dianov E M, Karpov V I, Grekov M V, Golant K M, Vasiliev S A, Medvekov O I and Khrapko R R 1997 Thermo-induced long period fibre grating IOOC-ECOC1997 vol 2 (London: IEE) pp 53-6

[16] Dianov E M, Karpov V I, Kurkov A S and Grekov M V 1998 Long period fibre gratings and mode-field converters fabricated by thermodiffusion in phosphosilicate fibres $24^{\text {th }}$ ECOC vols 1-3 (Madrid: Telefonica Espana) pp 395-6

[17] Kim C S, Han Y, Lee B H, Han W-T, Paek U-C and Chung Y 2000 Induction of the refractive index change in B-doped optical fibers through relaxation of the mechanicalstress Opt. Commun. $185337-42$ [18] Rego G, Okhotnikov O, Dianov E and Sulimov V 2001 Hightemperature stability of long-period fibre gratings using an electric arc $J$. Lightwave Technol

[ 19] 1574-9[19] Palai P, SatyanarayanMN, Das M, ThyagarajanK and Pal B P 2001 Characterisation and simulation of long period gratings fabricated using electric discharge Opt. Commun.193 181-5

[20] Savin S, Digonnet M J F, Kino G S and Shaw H J 2000 Tunable mechanically induced long-period fibre gratings Opt. Lett. 25 710-12 [21] Jiang Y, Li Q, Lin C H, Lyons E, Tomov I and Lee H P 2002 A novel strain-induced thermally tuned long-period fibre grating fabricated on a periodic corrugated silicon fixtureIEEE Photon. Technol. Lett. 14 $941-3$

[22] Kakarantzas G, Dimmick T E, Birks T A, Le Roux R and Russell P St J 2001 Miniature all-fibre devices based on CO2 microstructuring of apered fibres Opt. Lett. 26 1137-9

[23] Poole C D, Presby H M and Meester J P 1994 Two mode fibre spatial-mode converter using periodic core deformation Electron. Lett.30 $1437-8$

[24] Narayanan C, Presby H M and Vengsarkar A M 1996 Bandrejection fibre filter using periodic core deformation OFC 1996 (San Jose, USA) pp 267-8

25] Lin C-Y, Chern G-W andWang L A 2001 Periodical corrugated structure for forming sampled fibre Bragg grating and long-period fibre grating with tunable coupling strength J. Lightwave Technol. 19 1212-20

[26] Kakarantzas G, Birks T A and Russell P S 2002 Structural longperiod gratings in photonic crystal fibers Opt. Lett. 27 1013-15

[27] Kashyap R 1999 Fibre Bragg Gratings (London: Academic)

[28] Williams D L, Ainslie B J, Armitage J R, Kashyap R and Campbell R 1993 Enhanced UV photosensitivity in boron codoped germanosilicate fibers Electron. Lett.29 45-7

[29] Lemaire P J, Atkins R M, Mizrahiu V and ReedW A 1993 High pressure H2-loading as a technique for achieving ultrahigh UV photosensitivity and thermal sensitivity in $\mathrm{GeO} 2$ doped optical fibers Electron. Lett.29 1191-3

[30] Vengsarkar A M, Lemaire P J, Judkins J B, Bhatia V, Erdogan T and Sipe J E 1996 Long-period fibre gratings as band rejection filters $J$. Lightwave Technol. 14 58-64

[31] Patrick H J, Askins C G, Mcelhanon R W and Friebele E J 1997 Amplitude mask patterned on an excimer laser mirror for high intensity writing of long period fibre gratings

Electron.Lett.33 1167-8

[32] Lu S Y, Tan H Y and Demokan M S 1999 Low-cost microlens array for long-period grating fabrication Electron. Lett.35 79-80

[33] Ye C C, James S W and Tatam R P 2000 Simultaneous temperature and bend sensing using long-period fibre gratings Opt. Lett. 25 1007-9

[34] A Thesis Of Doctor Of Philosophy Of Nidhi, Department Of Electronics \& Communication Engineering Thapar University,Performance Analysis And Design Of Fibre Gratings For Sensing Applications.

[35] Coupled Mode Theory for Long Period Grating written in 980nm pumped Erbium Doped Fiber, Rashmi Singh and Enakshi K. Sharma, Department of Electronic Science,University of Delhi south campus, https://www.google.co.in/search?q=Rashmi+Singh\%2C+Sunanda+and+ E.K.Sharma\%2C+Gain+Flattening+by+long+period+gratings+in+erbiu $\mathrm{m}+$ doped+fibers $\% 2 \mathrm{C}+$ Optics+Communications $\% 2 \mathrm{C}+240+(2004)+123$ 